\title{
Hospital Readmissions After Surgical Treatment of Proximal Humerus Fractures: Is Arthroplasty Safer Than Open Reduction Internal Fixation?
}

\author{
Alan L. Zhang MD, William W. Schairer MD, \\ Brian T. Feeley MD
}

Received: 2 December 2013/Accepted: 27 March 2014/Published online: 15 April 2014

(C) The Association of Bone and Joint Surgeons (B) 2014

\begin{abstract}
Background With technologic advances such as locked periarticular plating, hemiarthroplasty of the humeral head, and more recently reverse total shoulder replacement, surgical treatment of proximal humerus fractures has become more commonplace. However, there is insufficient information regarding patient outcomes after surgery, such as the frequency of unplanned hospital readmissions and factors contributing to readmission.

Questions/purposes We measured (1) the frequency of unplanned hospital readmissions after surgical treatment of proximal humerus fractures, (2) the medical and surgical causes of readmission, and (3) the risk factors associated with unplanned readmissions.

Methods The State Inpatient Database from seven different states was used to identify patients who underwent treatment for a proximal humerus fracture with open reduction and internal fixation (ORIF), hemiarthroplasty of the humeral head, or reverse total shoulder arthroplasty from 2005 through 2010. The database was used to measure the 30-day and 90-day readmission rates and identify causes and risk factors for readmission. Multivariate
\end{abstract}

Each author certifies that he or she, or a member of his or her immediate family, has no funding or commercial associations (eg, consultancies, stock ownership, equity interest, patent/licensing arrangements, etc) that might pose a conflict of interest in connection with the submitted article.

All ICMJE Conflict of Interest Forms for authors and Clinical Orthopaedics and Related Research editors and board members are on file with the publication and can be viewed on request.

A. L. Zhang ( $₫)$, W. W. Schairer, B. T. Feeley

Department of Orthopaedic Surgery, University of California

San Francisco, 1500 Owens Street, Box 3004, San Francisco, CA 94158, USA

e-mail: ZhangA@orthosurg.ucsf.edu; azhangmd@gmail.com modeling and a Cox proportional hazards model were used for statistical analysis.

Results A total of 27,017 patients were included with an overall 90-day readmission rate of $14 \%$ (15\% for treatment with ORIF, $15 \%$ for reverse total shoulder arthroplasty, and $13 \%$ for hemiarthroplasty). The majority of readmissions were associated with medical diagnoses $(75 \%)$, but treatment with ORIF was associated with the most readmissions from surgical complications, (29\%) followed by reverse total shoulder arthroplasty (20\%) and hemiarthroplasty $(16 \%)$ ( $\mathrm{p}<0.001)$. Risk of readmission was greater for patients who were female, African American, discharged to a nursing facility, or had Medicaid insurance.

Conclusions As the majority of unplanned hospital readmissions were associated with medical diagnoses, it is important to consider patient medical comorbidities before surgical treatment of proximal humerus fractures and during the postoperative care phase.

Level of Evidence Level III, therapeutic study. See the Instructions for Authors for a complete description of levels of evidence.

\section{Introduction}

Although results of surgical treatment of proximal humerus fractures have improved with advancements in implants and surgical techniques, the proper treatment for these fractures remains controversial. This is partly the result of the reported complications associated with many of the surgical options. Complication rates have been reported as high with open reduction and internal fixation (ORIF) despite technologic improvements such as locked plating [14, 17, 18, 25, 31]. Although surgeons now are using hemiarthroplasty of the humeral head and reverse total 
shoulder replacement in an attempt to improve functional outcomes after treatment of comminuted three- or four-part fractures and fractures that have a head-split component $[3$, $6,10,15,23]$, these arthroplasty techniques for fracture care also have associated complications [3, 39].

Postoperative complications can result in unplanned hospital readmissions, which can result in harm to patients and increased healthcare costs. In 2004, nearly one in five hospitalized patients who was a Medicare beneficiary was readmitted within 30 days at an estimated annual cost of USD 17.4 billion [21]. New programs such as the Readmission Reduction Program have established metrics to reduce payments to hospitals with disproportionately high readmission rates [19]. Additionally, the Centers for Medicare \& Medicaid Services publish hospital readmission rates and other quality metrics [27]. These regulatory programs make identification of factors that lead to readmissions an important area for improvement in healthcare expense and outcomes [1]. We recently published a report describing the frequency and risk factors for hospital readmission for patients who had primary elective shoulder arthroplasty [34], but we believe it is important to validate and extend these findings in a population of patients treated for proximal humerus fractures. As the use of open treatment for shoulder fractures has increased, it is critical to evaluate the kinds of complications that can arise after surgical treatment of these injuries. The introduction of periarticular locked plating and shoulder arthroplasty have allowed for improved treatment of comminuted proximal humerus fractures. However, these remain complex and relatively new procedures with much to be understood about improving patient selection and indications while reducing complications.

We therefore sought to evaluate readmission rates within 90 days of surgical treatment for proximal humerus fractures and to describe the diagnoses leading to readmission and the associated risk factors. Specifically, we measured (1) the unplanned hospital readmission rate after surgical treatment of proximal humerus fractures, (2) the medical and surgical causes of readmission, and (3) the risk factors associated with unplanned readmissions.

\section{Materials and Methods}

The State Inpatient Database [36], produced by the Healthcare Cost and Utilization Project (HCUP) under the Agency for Healthcare Research and Quality was used to identify surgical procedures used to treat proximal humerus fractures. The database contains inpatient records from various states from 2005 through 2010. The states and years used in this analysis were selected based on the availability of unique identifiers to track patient visits with time and included California (2005-2010), Florida (20052010), Hawaii (2006-2007), North Carolina (2006-2010), Nebraska (2006-2010), New York (2006-2009), and Utah (2006-2009).

We recently performed a related study in the elective shoulder arthroplasty population [34]. Although the number of patients whose records were evaluated in that study was similar to those studied here $(26,218$ and 27,017 , respectively), and although some patients in this series were treated with arthroplasties, we are certain that no patient was counted twice; that is, there was no overlap of patient data between the two reports. We were able to ensure this by selecting for patients who had a unique ICD9 diagnosis code for proximal humerus fracture (812.00, $812.01,812.02,812.03,812.09,812.10,812.11,812.12$, $812.13,812.19$ ) for this study while only selecting for patients who had ICD-9 codes for arthritis or cuff tear arthropathy in the previous study. This way, patients who had a hemiarthroplasty or a total shoulder arthroplasty would be counted only in this study if the surgery was used to treat a proximal humerus fracture and not if it was used to treat arthritis or any other diagnosis.

Patients who underwent surgical treatment for proximal humerus fractures were tracked through the database to measure the 30-day and 90-day readmission rates and to identify the causes and risk factors associated with readmission within 90 days of surgery. Procedures evaluated in this study were the ORIF, hemiarthroplasty of the humeral head, and reverse total shoulder arthroplasty.

Procedures and diagnoses were identified by ICD-9 Clinical Modification (ICD-9-CM) and ICD-9 codes, respectively (Appendix 1). Although ORIF (79.31) and hemiarthroplasty of the humeral head (81.81) have unique codes, total shoulder arthroplasty and reverse total shoulder arthroplasty shared the same code (81.80) until fiscal year 2010 when reverse total shoulder arthroplasty was assigned a unique code (81.88). All total shoulder arthroplasty codes with an accompanying proximal humerus fracture diagnosis were assumed to be a reverse total shoulder arthroplasty because the majority are treated with a reverse total shoulder arthroplasty rather than a standard total shoulder arthroplasty (Fig. 1). We excluded any hemiarthroplasties or reverse total shoulder arthroplasties performed for arthritis or rotator cuff tear arthropathy and included only those performed for the treatment of proximal humerus fractures. Patients with polytrauma with concomitant diagnoses such as pneumothorax, hemothorax, cranial injury, or exploratory laparotomy also were excluded from this study. Finally, we excluded patients who did not have at least 90 days followup and who identified their home state as different from that of where they underwent surgery to ensure that all readmissions could be tracked accurately. 


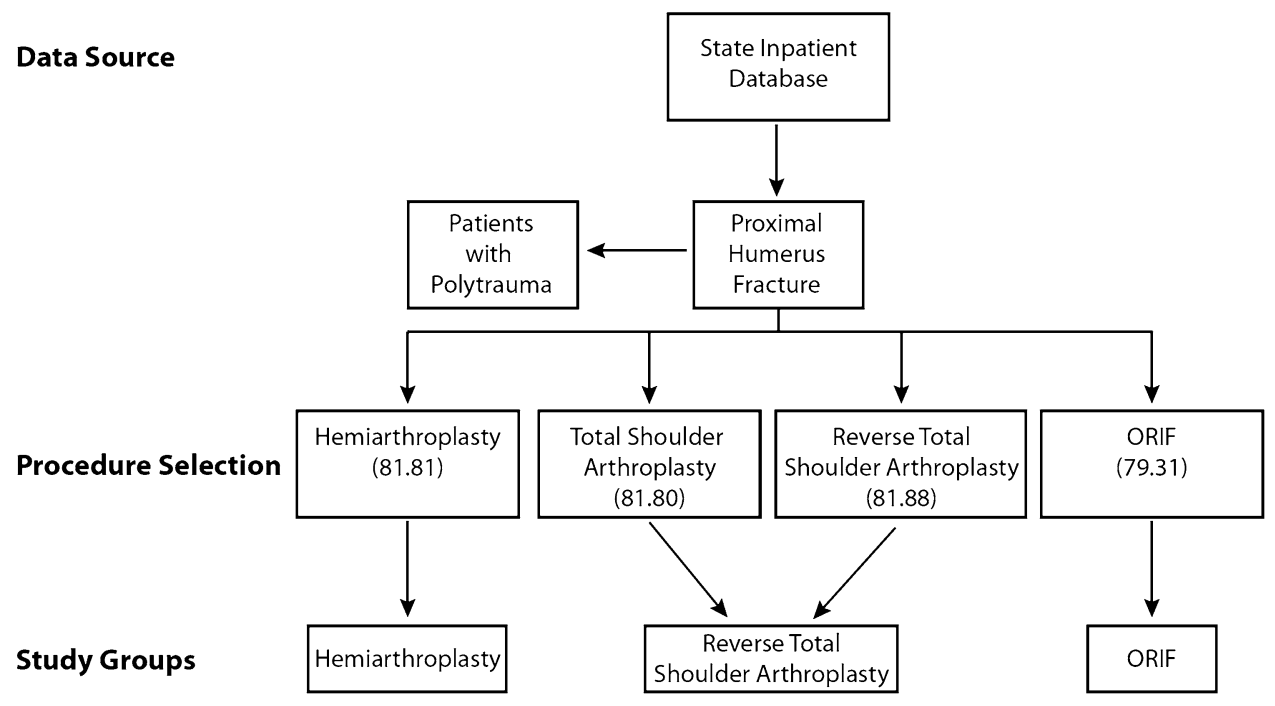

Fig. 1 Patients were selected from the State Inpatient Database using ICD-9 procedure codes. Proximal humerus fractures were selected after excluding patients with polytrauma and then stratified based on

Patient medical comorbidities were assessed using the definitions of Elixhauser et al. used for administrative claims data [11], which provides a comprehensive set of 30 comorbidity measures for use with large administrative inpatient datasets. Primary diagnoses at readmission were reviewed and grouped as surgical (directly attributable to the index procedure) or medical. Medical diagnoses were further grouped according to the Clinical Classifications Software as defined by the HCUP [16]. Hospital volume was calculated for each procedure and classified as low (less than one procedure per quarter), medium (less than one procedure per month), or high (greater than one procedure per month) [29].

Multivariate modeling was performed to evaluate factors associated with hospital readmission within 90 days. Risk was calculated using a Cox proportional hazards model from a time-to-readmission analysis. Variables included procedure, age, sex, hospital procedure volume, and medical comorbidities. Statistical significance was set at $\mathrm{p}$ less than 0.05 .

\section{Results}

Demographics

A total of 27,017 patients who were treated for a proximal humerus fracture were included in this study (Table 1). There were 18,392 patients who underwent ORIF for proximal humerus fractures, 7765 who underwent hemiarthroplasty of the numeral head, and 860 who underwent reverse total shoulder arthroplasty. treatment with open reduction internal fixation (ORIF), hemiarthroplasty of the humeral head, or reverse total shoulder arthroplasty.

Frequency of Readmission

The 30-day readmission rate was $8 \%$ and the overall 90 day readmission rate was $14 \%$ (3887 of 27,017). Further analysis revealed rates of $15 \%$ for ORIF and reverse total shoulder arthroplasty and $13 \%$ for hemiarthroplasty. Patients who underwent hemiarthroplasty had fewer readmissions compared with patients who had ORIF or reverse total shoulder arthroplasty $(\mathrm{p}<0.001)$ (Fig. 2). Furthermore, $29 \%$ of patients were readmitted to a different hospital than where the original surgery was performed. Patients who underwent ORIF at low-volume hospitals had a 90-day readmission rate of $16 \%$ compared with mediumvolume $(15 \%)$ and high-volume hospitals $(14 \%)(\mathrm{p}=$ 0.002). Patients who had hemiarthroplasties or reverse total shoulder arthroplasties showed no differences in readmission rates when their surgery was performed at low-, medium-, or high-volume hospitals $(\mathrm{p}=0.33$ and $\mathrm{p}=0.80$, respectively). A subgroup of 1378 patients were classified as patients with polytrauma and were excluded from the main arm of the study. In this polytrauma subgroup, the 30day readmission rate was $11 \%$ and the overall 90-day readmission rate was $20 \%$, which was higher than the rate for the main cohort $(\mathrm{p}<0.0001)$.

\section{Causes of Readmission}

The majority of readmissions were the result of medical complications (75\% [2901 of 3887]), whereas surgical complications contributed to $25 \%$ of total readmissions (Table 2). With respect to each procedure, ORIF was 
Table 1. Patient demographics

\begin{tabular}{|c|c|c|c|}
\hline Demographic & ORIF & $\begin{array}{l}\text { Hemiarthroplasty } \\
\text { of the humeral } \\
\text { head }\end{array}$ & $\begin{array}{l}\text { Reverse total } \\
\text { shoulder } \\
\text { arthroplasty }\end{array}$ \\
\hline Number of patients & 18,392 & 7765 & 860 \\
\hline Age (years \pm SD) & $65 \pm 18$ & $73 \pm 12$ & $75 \pm 11$ \\
\hline Female & $70 \%$ & $79 \%$ & $80 \%$ \\
\hline $\begin{array}{l}\text { Length of stay } \\
\quad \text { (days } \pm \text { SD) }\end{array}$ & $4.5 \pm 5.2$ & $4.8 \pm 4.5$ & $4.7 \pm 4.3$ \\
\hline $\begin{array}{l}\text { Total comorbidities } \\
\quad \text { (average } \pm \text { SD) }\end{array}$ & $2.0 \pm 1.7$ & $2.3 \pm 1.7$ & $2.4 \pm 1.6$ \\
\hline In-hospital mortality & $0.6 \%$ & $0.5 \%$ & $0.8 \%$ \\
\hline \multicolumn{4}{|l|}{ Insurance } \\
\hline Medicare & $54 \%$ & $72 \%$ & $78 \%$ \\
\hline Medicaid & $5.3 \%$ & $2.9 \%$ & $3.3 \%$ \\
\hline Private & $30 \%$ & $19 \%$ & $14 \%$ \\
\hline Self-pay & $3.5 \%$ & $1.4 \%$ & $1.1 \%$ \\
\hline Other & $7.1 \%$ & $4.5 \%$ & $3.6 \%$ \\
\hline \multicolumn{4}{|l|}{ Race } \\
\hline Caucasian & $82 \%$ & $86 \%$ & $87 \%$ \\
\hline African American & $3.7 \%$ & $1.6 \%$ & $1.4 \%$ \\
\hline Hispanic & $9.6 \%$ & $8.5 \%$ & $8.1 \%$ \\
\hline Asian & $2.4 \%$ & $1.6 \%$ & $1.6 \%$ \\
\hline Other & $2.6 \%$ & $2.5 \%$ & $1.6 \%$ \\
\hline
\end{tabular}

ORIF $=$ open reduction and internal fixation.

associated with the highest proportion of readmissions from surgical complications (29\%), followed by reverse total shoulder arthroplasty (20\%) and hemiarthroplasty (16\%) $(\mathrm{p}<0.001)$. Surgical readmissions after ORIF were most commonly associated with mechanical complications (11\% of all readmissions), followed by infection (7.5\%). For hemiarthroplasty, dislocation was the most common surgical diagnosis associated with readmission (4.5\%), followed by infection (4.3\%). In the reverse total shoulder arthroplasty group, dislocation also was the leading surgical diagnosis $(10 \%)$ compared with just $1 \%$ in the ORIF group $(\mathrm{p}<0.001)$. Infection was more common with ORIF (7.5\%) than with hemiarthroplasty $(4.3 \%)$ or reverse total shoulder arthroplasty $(2.4 \%)(\mathrm{p}<0.001)$. Septicemia was the primary medical diagnosis in $4.0 \%$ of readmissions after ORIF, $4.7 \%$ after hemiarthroplasty, and $4.0 \%$ after reverse total shoulder arthroplasty $(p>0.05)$. Other common medical causes of readmission included deep venous thrombosis or pulmonary embolus $(3.1 \%, 3.8 \%$, and $4.8 \%)$ and secondary hip fracture $(2.1 \%, 3.7 \%$, and $3.9 \%)$, respectively.

\section{Risk Factors for Readmission}

There was no difference in risk of readmission with older age (Table 3). Patients who had hemiarthroplasty ( $<<$

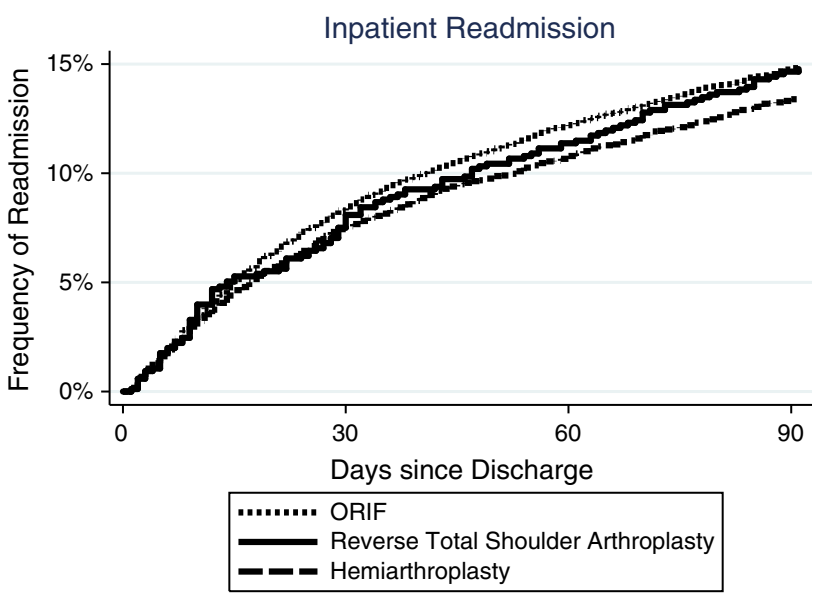

Fig. 2 The graph illustrates the frequency of hospital readmissions for patients with proximal humerus fractures treated by open reduction internal fixation (ORIF) compared with hemiarthroplasty and reverse total shoulder arthroplasty. Readmissions for hemiarthroplasty were less than for ORIF.

$0.001)$ and reverse total shoulder arthroplasty $(\mathrm{p}=0.04)$ showed a decreased risk of readmission compared with patients who had ORIF. Males had a $23 \%$ less risk of readmission than females, and when comparing different racial groups, African Americans had a greater risk of readmission than all other groups $(p=0.03)$. Patients with Medicaid insurance had more than a $27 \%$ greater risk of readmission compared with patients with Medicare, whereas patients with private insurance or who were selfpay had $18 \%$ and $9 \%$ lower risks, respectively, compared with patients with Medicare. Patients who were discharged to home with home health services had a $19 \%$ greater risk of readmission than patients who were discharged home without ancillary support, whereas patients who were transferred to a rehabilitation or nursing facility had nearly a twofold greater risk of readmission. There was no difference in risk of readmission for patients with procedures performed at low-, medium-, or high-volume hospitals. Finally, for each additional medical comorbidity as defined by the Elixhauser et al. [11], the risk of readmission increased by $20 \%$.

\section{Discussion}

With technologic advances such as locked plating, hemiarthroplasty, and reverse total shoulder arthroplasty, surgical treatment of proximal humerus fractures has become more commonplace. However, there is insufficient information regarding patient outcomes after surgery, such as the frequency of unplanned hospital readmissions and factors contributing to readmission. We therefore sought to evaluate readmission rates within 90 days of surgical 
Table 2. Causes of readmission after open treatment of proximal humerus fracture

\begin{tabular}{|c|c|}
\hline Cause of readmission & $\begin{array}{l}\text { Percent of readmission } \\
\text { (percent of procedures) }\end{array}$ \\
\hline \multicolumn{2}{|l|}{ ORIF for fracture } \\
\hline Surgical & $29(4.3)$ \\
\hline Mechanical complication & $11(1.7)$ \\
\hline Infection & $8(1.1)$ \\
\hline Humerus fracture & $4(0.6)$ \\
\hline Malunion/nonunion & $3(0.4)$ \\
\hline Dislocation & $1.0(0.1)$ \\
\hline Medical & $71(11)$ \\
\hline Septicemia & $4.0(0.6)$ \\
\hline Pneumonia & $3.1(0.5)$ \\
\hline $\begin{array}{l}\text { Deep vein thrombosis or } \\
\text { pulmonary embolism }\end{array}$ & $3.1(0.5)$ \\
\hline Congestive heart failure & $3.0(0.4)$ \\
\hline Urinary tract infections & $2.7(0.4)$ \\
\hline \multicolumn{2}{|l|}{ Hemiarthroplasty for fracture } \\
\hline Surgical & $16(2.2)$ \\
\hline Dislocation & $4.5(0.6)$ \\
\hline Infection & $4.3(0.6)$ \\
\hline Humerus fracture & $2.6(0.3)$ \\
\hline Mechanical complication & $1.7(0.2)$ \\
\hline Hematoma & $1.0(0.1)$ \\
\hline Medical & $84(11.2)$ \\
\hline Septicemia & $4.7(0.6)$ \\
\hline Pneumonia & $4.6(0.6)$ \\
\hline $\begin{array}{l}\text { Deep vein thrombosis or } \\
\text { pulmonary embolism }\end{array}$ & $3.8(0.5)$ \\
\hline Fracture of neck of femur (hip) & $3.7(0.5)$ \\
\hline Urinary tract infections & $3.7(0.5)$ \\
\hline \multicolumn{2}{|c|}{ Reverse total shoulder arthroplasty for fracture } \\
\hline Surgical & $20(2.9)$ \\
\hline Dislocation & $9.6(1.4)$ \\
\hline Humerus fracture & $3.2(0.5)$ \\
\hline Mechanical complication & $2.4(0.3)$ \\
\hline Infection & $2.4(0.3)$ \\
\hline Periprosthetic fracture & $1.6(0.2)$ \\
\hline Medical & $80(11.6)$ \\
\hline Congestive heart failure & $4.8(0.7)$ \\
\hline $\begin{array}{l}\text { Deep vein thrombosis or } \\
\text { pulmonary embolism }\end{array}$ & $4.8(0.7)$ \\
\hline Septicemia & $4.0(0.6)$ \\
\hline Gastrointestinal hemorrhage & $4.0(0.6)$ \\
\hline Nonspecific chest pain & $4.0(0.6)$ \\
\hline
\end{tabular}

Top five medical and surgical diagnoses of readmission after each procedure. Rates represented as a percentage of readmissions for that procedure and as a percentage of total cases performed for that procedure in parentheses; ORIF = open reduction and internal fixation.
Table 3. Risk factors for readmission after open treatment of proximal humerus fracture

\begin{tabular}{|c|c|c|c|}
\hline Variable & $\begin{array}{l}\text { Hazard } \\
\text { ratio }\end{array}$ & $95 \% \mathrm{CI}$ & $\mathrm{p}$ value \\
\hline \multicolumn{4}{|l|}{ Age (reference $<55$ years) } \\
\hline $55-74$ years & 0.97 & $0.86-1.09$ & 0.611 \\
\hline $75+$ years & 1.06 & $0.93-1.21$ & 0.413 \\
\hline \multicolumn{4}{|l|}{ Treatment type (reference ORIF) } \\
\hline Hemiarthroplasty & 0.77 & $0.71-0.83$ & $<0.001$ \\
\hline Reverse total shoulder arthroplasty & 0.82 & $0.67-0.99$ & 0.043 \\
\hline Male & 0.77 & $0.72-0.83$ & $<0.001$ \\
\hline \multicolumn{4}{|l|}{ Race (reference Caucasian) } \\
\hline African American & 1.22 & $1.02-1.46$ & 0.033 \\
\hline Asian & 1.00 & $0.89-1.12$ & 0.964 \\
\hline Hispanic & 0.89 & $0.70-1.15$ & 0.380 \\
\hline Other & 0.90 & $0.72-1.14$ & 0.395 \\
\hline \multicolumn{4}{|l|}{ Insurance (reference Medicare) } \\
\hline Medicaid & 1.27 & $1.08-1.49$ & 0.004 \\
\hline Private & 0.82 & $0.74-0.91$ & $<0.001$ \\
\hline Self-pay & 0.91 & $0.71-1.17$ & 0.474 \\
\hline Other & 0.76 & $0.63-0.92$ & 0.005 \\
\hline \multicolumn{4}{|l|}{ Discharge status (reference home) } \\
\hline Home with services & 1.19 & $1.07-1.32$ & 0.001 \\
\hline $\begin{array}{l}\text { Transfer to facility } \\
\text { (rehabilitation/nursing) }\end{array}$ & 1.99 & $1.82-2.18$ & $<0.001$ \\
\hline \multicolumn{4}{|l|}{ Hospital volume (reference low) } \\
\hline Medium & 0.99 & $0.91-1.07$ & 0.733 \\
\hline High & 0.93 & $0.84-1.02$ & 0.139 \\
\hline Medical comorbidities (per diagnosis) & 1.20 & $1.18-1.22$ & $<0.001$ \\
\hline
\end{tabular}

Risk factors for readmission after open treatment of proximal humerus fractures represented as hazard ratios. Statistical significance set for $\mathrm{p}<0.05$; ORIF $=$ open reduction and internal fixation.

treatment for proximal humerus fractures and to describe the diagnoses leading to readmission and the associated risk factors through a large, multistate database. In doing so, we present novel information showing that although the majority of readmissions were associated with medical complications, treatment with ORIF was associated with the highest proportion of readmissions from surgical complications, followed by reverse total shoulder arthroplasty and hemiarthroplasty.

Limitations of this study arise from the nature of administrative claims data, which do not contain clinical information such as details of the operation, training of the surgeon, complexity of the procedure, mechanism of injury, instrumentation used, or postoperative rehabilitation, yet the large sample size of these database analyses offers the ability to elicit trends that otherwise would be underpowered by clinical studies. A database analysis also may give 
rise to selection bias and transfer bias. In this study, we cannot ascertain if the original injury pattern was the same for each treatment group or the surgeon's indications for which surgical procedure was performed, but we were able to outline demographic information from the database (Table 1) which affords a comparison of age, gender, and other factors between each treatment group. Our results reflect the association of medial or surgical diagnoses with unplanned hospital readmissions and not causation. We cannot conclude that the surgical treatment directly caused the unplanned readmission as the initial injury could have influenced readmission but our aim is to report any associations. Another limitation is the lack of a unique procedure code for reverse total shoulder arthroplasty until recently. We assumed all total shoulder arthroplasty codes performed for proximal humerus fractures were reverse total shoulder arthroplasties before 2010 because there were no sources found that advocate for the use of a total shoulder arthroplasty for fracture. However, despite the lack of evidence in the literature, it is possible that a total shoulder arthroplasty is used because of surgeon preference and experience and thus incorrectly grouped with reverse total shoulder arthroplasties in our study. However, this frequency is assumed to be low given the general position in the literature. Finally, the information in the State Inpatient Database is limited to states that elect to participate and by whether they allow unique identifiers to track patients with time, which may create possible further bias. Using data from seven different states across the United States may help to minimize regional bias and create a better representation of the country, although these results should not be extrapolated as a comprehensive national analysis.

Our results showed that the overall readmission rate was high (14\%), and treatment with hemiarthroplasty resulted in a slightly lower readmission rate than treatment with ORIF or reverse total shoulder arthroplasty. To our knowledge, there have been no comparable studies investigating the frequency of hospital readmissions after surgical treatment of shoulder fractures. The closest study is our recent evaluation of hospital readmissions after primary shoulder arthroplasty [34]. We found that patients undergoing elective reverse total shoulder arthroplasty had the highest 90 -day readmission rate $(11 \%)$ followed by hemiarthroplasty (8\%) and total shoulder arthroplasty (6\%) with medical complications contributing to $82 \%$ of readmissions. Other similar studies that evaluated readmissions after shoulder arthroplasty include a single-state analysis of total shoulder arthroplasty that found a 60-day readmission rate of $6.7 \%$ [29], and a study from the veteran population showing 2-week readmissions to be $5.6 \%$ [12].

In contrast to previous studies advocating improved clinical results with the use of ORIF instead of arthroplasty procedures [8, 37], our results show that treatment of proximal humerus fractures with ORIF was associated with a higher proportion of readmissions from surgical complications $(29 \%)$ than reverse total shoulder arthroplasty $(20 \%)$ or hemiarthroplasty (16\%). Mechanical complications $(11 \%)$ was the most common surgical cause of readmission after ORIF which is consistent with reports indicating that even with the use of periarticular locked plating, screw cutout after ORIF is still one of the most common causes of surgical failure [24, 35]. Although shoulder arthroplasty components have been revised and advancements in techniques have shown improved clinical outcomes [32], our results indicate that instability after hemiarthroplasty or reverse total shoulder arthroplasty remains a concern. In the current study and in our study of the elective shoulder arthroplasty population [34], we found that dislocation is one of the leading causes of hospital readmission after shoulder arthroplasty procedures. In the current analysis, the most common surgical diagnosis associated with readmission after hemiarthroplasties and reverse total shoulder arthroplasties was dislocation $(4.5 \%$ and $10 \%$, respectively). For hemiarthroplasties, this dislocation rate has been attributed to failure of the greater tuberosity repair and was reported to be as much as $14 \%$ [13]. This led to the introduction of reverse total shoulder arthroplasty for the treatment of proximal humerus fractures with significant comminution of the greater tuberosity [23, 28]. The frequency of reverse total shoulder arthroplasty dislocations has been variable, ranging from $2.3 \%$ in patients with proximal humerus fractures [4] to $30.8 \%$ in patients after tumor resection [9]. A retrospective study of 240 reverse total shoulder arthroplasties found dislocation to be the most common complication with a frequency of $7.5 \%$ [38]. Because the reverse total shoulder arthroplasty is a relatively new procedure in the United States, it will be important to track the complication rates of this procedure as it relates to different diagnoses including cuff tear arthropathy, revision shoulder arthroplasty, and fractures. In terms of medical conditions associated with readmission, septicemia was the most common followed by deep vein thrombosis or pulmonary embolism. Our analysis showed that less than $1 \%$ of surgical procedures of the shoulder are associated with readmission from deep vein thrombosis or pulmonary embolism, which is consistent with the rate reported in a recent review [33]. A second fragility fracture in the form of a hip fracture is one of the more common reasons for readmission during the first 90 days after surgery for a proximal humerus fracture. This is consistent with a previous report that patients who have a proximal humerus fracture are at increased risk for a subsequent hip fracture the first year after injury [7].

We found that male patients had a lower risk for readmission than female patients after surgery for a proximal 
humerus fracture. This is contrary to our multivariate analysis [34] which showed males have higher risk for readmission after elective arthroplasty. This most likely was attributable to the increased risk for osteopenia and osteoporosis in a female cohort with proximal humerus fractures, as previous studies have shown a greater risk of surgical complications associated with decreased bone marrow density $[5,26]$. A higher risk of readmission after discharge to a skilled nursing facility found in both studies also reflects the medical condition of the patient. Healthier patients with less medical comorbidities are more likely to be discharged home instead of to a nursing facility after surgery and consequently have a lower risk of unplanned readmission. Patients with Medicaid insurance had more than a $27 \%$ increased risk of hospital readmission compared with patients with Medicare. This is a similar finding to our recent study [34] and an analysis by the HCUP that showed patients with Medicaid are $70 \%$ more likely to be readmitted than patients with private insurance [22]. It has been suggested that increased rates of complications in patients with Medicaid insurance result from less access to care and more complex clinical presentations compared with patients with private insurance [30]. Finally, procedural volume was found to affect readmission rates only for patients who had ORIF. Ninety-day readmission rates were significantly higher at low-volume centers than medium- and high-volume centers. Our findings in primary shoulder arthroplasty [34] and previous studies of hip and knee arthroplasties and one study of proximal humerus fractures showed decreased readmissions and complications in high-volume centers, which further supports the notion that surgeons who do not perform shoulder ORIF on a regular basis may have a higher complication or readmission rate $[2,20]$. Hemiarthoplasty and reverse total shoulder arthroplasty did not show a significant trend between different volume hospitals likely because of the smaller sample size in these two groups.

To our knowledge, our study includes the largest sample size with analysis of trends from multiple states and incorporates a complete analysis of frequency of hospital readmissions and associated diagnoses for 90 days from surgical treatment of proximal humerus fractures. We found that treatment with ORIF was associated with the greatest proportion of patients with surgical complications needing readmission compared with patients who had reverse total shoulder arthroplasty or hemiarthroplasty. In addition, as the majority of unplanned readmissions were associated with medical diagnoses, it is important to consider patients' medical comorbidities before surgery and during the postoperative care phase to help reduce the risk of hospital readmission.

\section{Appendix 1. ICD-9 diagnosis and procedure codes}

\begin{tabular}{ll}
\hline Diagnosis & ICD-9 diagnosis codes \\
\hline Proximal humerus fracture & 812.00812 .01812 .02812 .03812 .09 \\
& $\quad 812.10812 .11812 .12812 .13812 .19$ \\
Procedure & ICD-9 procedure codes \\
ORIF proximal humerus & 79.31 \\
Shoulder hemiarthroplasty & 81.81 \\
Total shoulder arthroplasty & 81.80 \\
Reverse total shoulder & 81.88 \\
$\quad$ &
\end{tabular}

ORIF = open reduction and internal fixation.

\section{References}

1. Bozic KJ. Value-based healthcare and orthopaedic surgery. Clin Orthop Relat Res: 2012;470:1004-1005.

2. Bozic KJ, Maselli J, Pekow PS, Lindenauer PK, Vail TP, Auerbach $\mathrm{AD}$. The influence of procedure volumes and standardization of care on quality and efficiency in total joint replacement surgery. $J$ Bone Joint Surg Am. 2010;92:2643-2652.

3. Brorson S, Rasmussen JV, Olsen BS, Frich LH, Jensen SL, Hróbjartsson A. Reverse shoulder arthroplasty in acute fractures of the proximal humerus: a systematic review. Int $J$ Shoulder Surg. 2013;7:70-78.

4. Bufquin T, Hersan A, Hubert L, Massin P. Reverse shoulder arthroplasty for the treatment of three- and four-part fractures of the proximal humerus in the elderly: a prospective review of 43 cases with a short-term follow-up. J Bone Joint Surg Br. 2007; 89:516-520.

5. Burke NG, Kennedy J, Green C, Dodds MK, Mullett H. Locking plate fixation for proximal humerus fractures. Orthopedics. 2012; 35:e250-254.

6. Chambers L, Dines JS, Lorich DG, Dines DM. Hemiarthroplasty for proximal humerus fractures. Curr Rev Musculoskelet Med. 2013;6: $57-62$.

7. Clinton J, Franta A, Polissar NL, Neradilek B, Mounce D, Fink HA, Schousboe JT, Matsen FA 3rd. Proximal humeral fracture as a risk factor for subsequent hip fractures. J Bone Joint Surg Am. 2009;91:503-511.

8. Dai J, Chai Y, Wang C, Wen G. Meta-analysis comparing locking plate fixation with hemiarthroplasty for complex proximal humeral fractures. Eur J Orthop Surg Traumatol. 2013 Feb 7 [Epub ahead of print].

9. De Wilde L, Sys G, Julien Y, Van Ovost E, Poffyn B, Trouilloud $P$. The reversed Delta shoulder prosthesis in reconstruction of the proximal humerus after tumour resection. Acta Orthop Belg. 2003;69:495-500.

10. Drake GN, O'Connor DP, Edwards TB. Indications for reverse total shoulder arthroplasty in rotator cuff disease. Clin Orthop Relat Res. 2010;468:1526-1533.

11. Elixhauser A, Steiner C, Harris DR, Coffey RM. Comorbidity measures for use with administrative data. Med Care. 1998;36: $8-27$.

12. Fehringer EV, Mikuls TR, Michaud KD, Henderson WG, O’Dell JR. Shoulder arthroplasties have fewer complications than hip or knee arthroplasties in US veterans. Clin Orthop Relat Res. 2010; 468:717-722.

13. Fucentese SF, Sutter R, Wolfensperger F, Jost B, Gerber C. Large metaphyseal volume hemiprostheses for complex fractures 
of the proximal humerus. J Shoulder Elbow Surg. 2014;23: 427-433.

14. Gregory TM, Vandenbussche E, Augereau B. Surgical treatment of three and four-part proximal humeral fractures. Orthop Traumatol Surg Res. 2013;99(1 suppl):S197-207.

15. Greiwe RM, Vargas-Ariza R, Bigliani LU, Levine WN, Ahmad CS. Hemiarthroplasty for head-split fractures of the proximal humerus. Orthopedics. 2013;36:e905-911.

16. HCUP. Healthcare Cost and Utilization Project. Clinical Classifications Software (CCS) for ICD-9-CM Fact Sheet. Available at: http://www.hcup-us.ahrq.gov/toolssoftware/ccs/ccsfactsheet.jsp. Accessed December 1, 2013.

17. Helmy N, Hintermann B. New trends in the treatment of proximal humerus fractures. Clin Orthop Relat Res. 2006;442:100-108.

18. Hirschmann MT, Quarz V, Audigé L. Internal fixation of unstable proximal humerus fractures with an anatomically preshaped interlocking plate: a clinical and radiologic evaluation. J Trauma. 2007;63:1314-1323.

19. Hospital Readmissions Reduction Program. The Patient Protection and Affordable Care Act. HR 3590, Sect. 3025. Available at: http://www.gpo.gov/fdsys/pkg/PLAW-111publ148/pdf/PLAW111publ148.pdf. Accessed March 14, 2014.

20. Jain NB, Kuye I, Higgins LD, Warner JJ. Surgeon volume is associated with cost and variation in surgical treatment of proximal humeral fractures. Clin Orthop Relat Res. 2013; 471:655-664.

21. Jencks SF, Williams MV, Coleman EA. Rehospitalizations among patients in the Medicare fee-for-service program. $N$ Engl $J$ Med. 2009;360:1418-1428.

22. Jiang HJ, Wier LM. All-cause hospital readmissions among nonelderly Medicaid patients, 2007. HCUP Statistical Brief \#89. April 2010. Agency for Healthcare Research and Quality, Rockville, MD. Available at: http://www.hcupus.ahrq.gov/ reports/statbriefs/sb89.pdf. Accessed December 1, 2013.

23. Jones KJ, Dines DM, Gulotta L, Dines JS. Management of proximal humerus fractures utilizing reverse total shoulder arthroplasty. Curr Rev Musculoskelet Med. 2013;6:63-70.

24. Jost B, Spross C, Grehn H, Gerber C. Locking plate fixation of fractures of the proximal humerus: analysis of complications, revision strategies and outcome. J Shoulder Elbow Surg. 2013;22:542-549.

25. Koukakis A, Apostolou CD, Taneja T, Korres DS, Amini A. Fixation of proximal humerus fractures using the PHILOS plate: early experience. Clin Orthop Relat Res. 2006;442:115-120.

26. Krappinger D, Bizzotto N, Riedmann S, Kammerlander C, Hengg C, Kralinger FS. Predicting failure after surgical fixation of proximal humerus fractures. Injury. 2011;42:1283-1288.
27. Kurtz S, Mowat F, Ong K, Chan N, Lau E, Halpern M. Prevalence of primary and revision total hip and knee arthroplasty in the United States from 1990 through 2002. J Bone Joint Surg Am. 2005;87:1487-1497.

28. Lenarz C, Shishani Y, McCrum C, Nowinski RJ, Edwards TB, Gobezie R. Is reverse shoulder arthroplasty appropriate for the treatment of fractures in the older patient? Early observations. Clin Orthop Relat Res. 2011;469:3324-3331.

29. Lyman S, Jones EC, Bach PB, Peterson MG, Marx RG. The association between hospital volume and total shoulder arthroplasty outcomes. Clin Orthop Relat Res. 2005;432:132-137.

30. Martin CT, Callaghan JJ, Liu SS, Gao Y, Warth LC, Johnston RC. Disparity in total joint arthroplasty patient comorbidities, demographics, and postoperative outcomes based on insurance payer type. J Arthroplasty. 2012;27:1761-1765.e1.

31. Moonot P, Ashwood N, Hamlet M. Early results for treatment of three and four-part fractures of the proximal humerus using the PHILOS plate system. J Bone Joint Surg Br. 2007;89:1206-1209.

32. Namdari S, Horneff JG, Baldwin K. Comparison of hemiarthroplasty and reverse arthroplasty for treatment of proximal humeral fractures: a systematic review. J Bone Joint Surg Am. 2013;95: 1701-1708.

33. Saleh HE, Pennings AL, Elmaraghy AW. Venous thromboembolism after shoulder arthroplasty: a systematic review. J Shoulder Elbow Surg. 2013;22:1440-1448.

34. Schairer WW, Zhang AL, Feeley BT. Hospital readmissions after primary shoulder arthroplasty. J Shoulder Elbow Surg. 2014 Mar 4. [Epub ahead of print]

35. Schliemann B, Siemoneit J, Theisen Ch, Kösters C, Weimann A, Raschke MJ. Complex fractures of the proximal humerus in the elderly: outcome and complications after locking plate fixation. Musculoskelet Surg. 2012;96(suppl 1):S3-11.

36. State Inpatient Database (SID) by the Healthcare Cost and Utilization Project. Available at: http://www.hcup-us.ahrq.gov/ sidoverview.jsp. Accessed March 24, 2014.

37. Südkamp N, Bayer J, Hepp P, Voigt C, Oestern H, Kääb M, Luo C, Plecko M, Wendt K, Köstler W, Konrad G. Open reduction and internal fixation of proximal humeral fractures with use of the locking proximal humerus plate: results of a prospective, multicenter, observational study. J Bone Joint Surg Am. 2009;91: $1320-1328$

38. Wall B, Nove-Josserand L, O'Connor DP, Edwards TB, Walch G. Reverse total shoulder arthroplasty: a review of results according to etiology. J Bone Joint Surg Am. 2007;89:1476-1485.

39. Wiesel BB, Nagda S, Williams GR. Technical pitfalls of shoulder hemiarthroplasty for fracture management. Orthop Clin North Am. 2013;44:317-329, viii. 